\title{
BFKL equation and anomalous dimensions in $N=4$ SUSY
}

\section{L.N. Lipatov}

Universität Hamburg, Germany

Petersburg Nuclear Physics Institute, Russia

\begin{abstract}
The BFKL approach to the Regge processes in QCD is reviewed. It is shown, that in the multi-colour QCD the BKP equations for composite states of several Reggeized gluons are integrable. We discuss the relation between Pomeron and Graviton in $\mathrm{N}=4$ SUSY. The property of the maximal transcendentality is formulated. It gives us a possibility to calculate the three-loop anomalous dimension. With the use of the asymptotic Bethe ansatz and a model for wrapping effects the anomalous dimension is calculated in four loops in an agreement with the BFKL and double-logarithmic predictions.
\end{abstract}

\section{Introduction}

It is known [1], that the QCD scattering amplitude in the leading logarithmic approximation (LLA) has the Regge-type asymptotics

$$
M_{A B}^{A^{\prime} B^{\prime}}(s, t)=\left.M_{A B}^{A^{\prime} B^{\prime}}(s, t)\right|_{B o r n} s^{\omega(t)},
$$

where $\left.M_{A B}^{A^{\prime} B^{\prime}}(s, t)\right|_{B o r n}$ is the Born amplitude and the gluon Regge trajectory is given below

$$
\omega\left(-|q|^{2}\right)=-\frac{\alpha_{c}}{4 \pi^{2}} N_{c} \int d^{2} k \frac{|q|^{2}}{|k|^{2}|q-k|^{2}} \approx-\frac{\alpha_{c}}{2 \pi} \ln \frac{\left|q^{2}\right|}{\lambda^{2}} .
$$

At high energies the gluons are produced in the multi-Regge kinematics. The elastic amplitude with the vacuum quantum numbers in the $t$-channel can be obtained with the use of the $s$-channel unitarity by summing over multi-gluon intermediate states [1]. It is convenient to introduce the complex variables for the gluon transverse coordinates and momenta

$$
\rho_{k}=x_{k}+i y_{k}, \rho_{k}^{*}=x_{k}-i y_{k}, p_{k}=i \frac{\partial}{\partial \rho_{k}}, p_{k}^{*}=i \frac{\partial}{\partial \rho_{k}^{*}} .
$$

In the coordinate representation the Balitsky-Fadin-Kuraev-Lipatov (BFKL) equation for the Pomeron wave function can be written as follows [1]

$$
E \Psi\left(\vec{\rho}_{1}, \vec{\rho}_{2}\right)=H_{12} \Psi\left(\vec{\rho}_{1}, \vec{\rho}_{2}\right), \Delta=-\frac{\alpha_{s} N_{c}}{2 \pi} \min E,
$$

where $\Delta$ is the Pomeron intercept. The BFKL Hamiltonian is presented below [2]

$$
H_{12}=\ln \left|p_{1} p_{2}\right|^{2}+\frac{1}{p_{1} p_{2}^{*}} \ln \left|\rho_{12}\right|^{2} p_{1} p_{2}^{*}+\frac{1}{p_{1}^{*} p_{2}} \ln \left|\rho_{12}\right|^{2} p_{1}^{*} p_{2}-4 \psi(1),
$$


where $\rho_{12}=\rho_{1}-\rho_{2}$. The kinetic energy is proportional to the gluon Regge trajectories, and the potential energy $\sim \ln \left|\rho_{12}\right|^{2}$ is related to the gluon production effective vertices.

The Hamiltonian is invariant under the Möbius transformation [3]

$$
\rho_{k} \rightarrow \frac{a \rho_{k}+b}{c \rho_{k}+d},
$$

where $a, b, c$ and $d$ are complex numbers. The eigenvalues of the Casimir operators

$$
M^{2}=\left(\sum_{r=1}^{2} \vec{M}^{(r)}\right)^{2}=\rho_{12}^{2} p_{1} p_{2}, M^{* 2}=\left(M^{2}\right)^{*}
$$

are related with the conformal weights

$$
m=1 / 2+i \nu+n / 2, \widetilde{m}=1 / 2+i \nu-n / 2
$$

for the principal series of unitary representations.

\section{Integrability of the BFKL dynamics at $N_{c} \rightarrow \infty$}

The Bartels-Kwiecinskii-Praszalowicz (BKP) equation [5] for the $n$-gluon composite state is simple at $N_{c} \rightarrow \infty$, where its Hamiltonian has the property of the holomorphic separability [4]

$$
H=\frac{1}{2} \sum_{k} H_{k, k+1}=\frac{1}{2}(h+h *),[h, h *]=0 .
$$

The holomorphic Hamiltonian can be written as follows

$$
h=\sum_{k} h_{k, k+1}, h_{12}=\ln \left(p_{1} p_{2}\right)+\frac{1}{p_{1}} \ln \rho_{12} p_{1}+\frac{1}{p_{2}} \ln \rho_{12} p_{2}-2 \psi(1),
$$

where $\psi(x)=(\ln \Gamma(x))^{\prime}$. As a result, we obtain for $\Psi$ the holomorphic factorization [4]

$$
\Psi\left(\vec{\rho}_{1}, \vec{\rho}_{2}, \ldots, \vec{\rho}_{n}\right)=\sum_{r, s} a_{r, s} \Psi_{r}\left(\rho_{1}, \ldots, \rho_{n}\right) \Psi_{s}\left(\rho_{1}^{*}, \ldots, \rho_{n}^{*}\right)
$$

and the duality symmetry [6]

$$
\rho_{r, r+1} \rightarrow p_{r} \rightarrow \rho_{r-1, r} .
$$

Moreover, there are integrals of motion commuting among themselves and with $h[2,7]$

$$
q_{r}=\sum_{k_{1}<k_{2}<\ldots<k_{r}} \rho_{k_{1} k_{2}} \rho_{k_{2} k_{3} \ldots \rho_{k_{r} k_{1}}} p_{k_{1}} p_{k_{2}} \ldots p_{k_{r}},\left[q_{r}, h\right]=0 .
$$

The integrability of the BFKL dynamics [7] is related to the fact, that $H$ coincides with the local Hamiltonian of the Heisenberg spin model [8].

In particular the Pomeron intercept $\Delta$ is positive [1]

$$
\Delta=4 \frac{\alpha_{s}}{\pi} N_{c} \ln 2
$$

and the Froissart bound for the total cross-section is violated. To restore the $s$-channel unitarity of scattering amplitudes one can use the effective field theory for Reggeized gluons [9]- [11]. 


\section{DGLAP and BFKL dynamics in $N=4$ SUSY}

The momenta $f_{a}\left(j, Q^{2}\right)$ of parton distributions satisfy the renormalization group equation with the anomalous dimension matrix $\gamma_{a b}$

$$
\frac{d}{d \ln Q^{2}} f_{a}\left(j, Q^{2}\right)=\sum_{b} \gamma_{a b}(j) f_{b}\left(j, Q^{2}\right)
$$

They are proportional to matrix elements of the light-cone components of twist- 2 operators

$$
O^{a}=\tilde{n}^{\mu_{1}} \ldots \tilde{n}^{\mu_{j}} O_{\mu_{1}, \ldots, \mu_{j}}^{a}, \tilde{\mathcal{O}}^{a}=\tilde{n}^{\mu_{1}} \ldots \tilde{n}^{\mu_{j}} \tilde{\mathcal{O}}_{\mu_{1}, \ldots, \mu_{j}}^{a} .
$$

The anomalous dimensions do not depend on other possible tensor projections

$$
\tilde{n}^{\mu_{1}} \ldots \tilde{n}^{\mu_{1+\omega}} O_{\mu_{1}, \ldots, \mu_{1+\omega}, \sigma_{1}, \ldots, \sigma_{|n|}}^{a} l_{\perp}^{\sigma_{1}} \ldots l_{\perp}^{\sigma_{|n|}} .
$$

The solution of the BFKL equation due to its Möbius invariance is classified by the anomalous dimension $\gamma=\frac{1}{2}+i \nu$ and the conformal spin $|n|$ coinciding with the number of transverse indices of $O$. The eigenvalue of the BFKL kernel in the next-to-leading approximation is

$$
\omega=\omega_{0}(n, \gamma)+4 \hat{a}^{2} \Delta(n, \gamma), \hat{a}=g^{2} N_{c} /\left(16 \pi^{2}\right) .
$$

In QCD $\Delta(n, \gamma)$ is a non-analytic function of the conformal spin $|n|[12,13]$

$$
\Delta_{Q C D}(n, \gamma)=c_{0} \delta_{n, 0}+c_{2} \delta_{n, 2}+\text { analytic terms },
$$

but in $N=4$ SUSY the Kroniker symbols are cancelled [13].

Moreover, in this model we obtain for $\Delta(n, \gamma)$ the Hermitian separability

$$
\begin{gathered}
\Delta(n, \gamma)=\phi(M)+\phi\left(M^{*}\right)-\frac{\rho(M)+\rho\left(M^{*}\right)}{2 \hat{a} / \omega}, M=\gamma+\frac{|n|}{2}, \\
\rho(M)=\beta^{\prime}(M)+\frac{1}{2} \zeta(2), \beta^{\prime}(z)=\frac{1}{4}\left[\Psi^{\prime}\left(\frac{z+1}{2}\right)-\Psi^{\prime}\left(\frac{z}{2}\right)\right] .
\end{gathered}
$$

It is important, that here all special functions have the maximal trancendentality property [13].

$$
\phi(M)=3 \zeta(3)+\Psi^{\prime \prime}(M)-2 \Phi(M)+2 \beta^{\prime}(M)(\Psi(1)-\Psi(M)),
$$

where

$$
\Phi(M)=\sum_{k=0}^{\infty} \frac{\beta^{\prime}(k+1)}{k+M}+\sum_{k=0}^{\infty} \frac{(-1)^{k}}{k+M}\left(\Psi^{\prime}(k+1)-\frac{\Psi(k+1)-\Psi(1)}{k+M}\right) .
$$

For the one loop anomalous dimension matrix of the twist-2 operators in the case $N=4$ the calculations were performed in Ref. [14]. The eigenvalues of this matrix are expressed in terms of the universal anomalous dimension for the super-multiplet unifying all twist-2 operators

$$
\gamma_{u n i}^{(0)}(j)=-4 S_{1}(j-2), S_{r}(j)=\sum_{i=1}^{j} \frac{1}{i^{r}} .
$$

Note, that $\gamma_{u n i}^{(0)}(j)$ has the maximal transcendentality property, which leads to an integrability of evolution equations for matrix elements of quasi-partonic operators in $N=4$ SUSY [14]. 


\section{Relation between Pomeron and Graviton}

The Pomeron intercept in the $N=4$ supersymmetric gauge theory was calculated recently at large coupling constants [17]. Here we shall review the basic arguments. To begin with, one can simplify the eigenvalue for the BFKL kernel in the diffusion approximation as follows (see [12])

$$
j=2-\Delta-D \nu^{2}, \gamma_{u n i}=\frac{j}{2}+i \nu,
$$

assuming, that the parameter $\Delta$ is small at large $z=\alpha N_{c} / \pi$. Due to the energy-momentum conservation we have $\left.\gamma\right|_{j=2}=0$ and therefore $\gamma$ can be expressed in terms of the parameter $\Delta$

$$
\gamma=(j-2)\left(\frac{1}{2}-\frac{1 / \Delta}{1+\sqrt{1+(j-2) / \Delta}}\right) .
$$

On the other hand with the use of the AdS/CFT correspondence [18] the above BFKL equation can be written as the graviton Regge trajectory

$$
j=2+\frac{\alpha^{\prime}}{2} t, t=E^{2} / R^{2}, \alpha^{\prime}=\frac{R^{2}}{2} \Delta .
$$

The behaviour of $\gamma$ at $g \rightarrow \infty, j \rightarrow \infty$ was predicted earlier [19]

$$
\gamma_{\mid z \rightarrow \infty}=-\sqrt{j-2} \Delta_{\mid j \rightarrow \infty}^{-1 / 2}=\sqrt{\pi j} z^{1 / 4} .
$$

Therefore one can calculate the Pomeron intercept at large coplings [17] (see also Ref. [23])

$$
j=2-\Delta, \Delta=\frac{1}{\pi} z^{-1 / 2} .
$$

\section{Two and three loops anomalous dimensions in $N=4$}

One can argue [13], that the perturbative expansion of the universal anomalous dimension

$$
\gamma_{u n i}(j)=\hat{a} \gamma_{u n i}^{(0)}(j)+\hat{a}^{2} \gamma_{u n i}^{(1)}(j)+\hat{a}^{3} \gamma_{u n i}^{(2)}(j)+\ldots
$$

contains in each order of the perturbation theory only special functions with the highest trancendentality. With such assumption one can obtain [13]

$$
\frac{1}{8} \gamma_{u n i}^{(1)}(j+2)=2 S_{1}(j)\left(S_{2}(j)+S_{-2}(j)\right)-2 S_{-2,1}(j)+S_{3}(j)+S_{-3}(j),
$$

where the corresponding harmonic sums are given below

$$
\begin{gathered}
S_{a}(j)=\sum_{m=1}^{j} \frac{1}{m^{a}}, S_{a, b, c, \cdots}(j)=\sum_{m=1}^{j} \frac{1}{m^{a}} S_{b, c, \cdots}(m), \\
S_{-a}(j)=\sum_{m=1}^{j} \frac{(-1)^{m}}{m^{a}}, S_{-a, b, \cdots}(j)=\sum_{m=1}^{j} \frac{(-1)^{m}}{m^{a}} S_{b, \cdots}(m),
\end{gathered}
$$




$$
\bar{S}_{-a, b, c \cdots(j)}=(-1)^{j} S_{-a, b, \ldots}(j)+S_{-a, b, \cdots}(\infty)\left(1-(-1)^{j}\right) .
$$

This result was verified by direct calculations of the anomalous dimension matrix in two loops [15].

Later the three-loop anomalous dimension matrix for QCD was calculated [16], which allowed us to find the universal anomalous dimension in three loops for $N=4$ SUSY [17]

$$
\begin{gathered}
\frac{1}{32} \gamma_{u n i}^{(2)}(j+2)=24 S_{-2,1,1,1}-12\left(S_{-3,1,1}+S_{-2,1,2}+S_{-2,2,1}\right) \\
+6\left(S_{-4,1}+S_{-3,2}+S_{-2,3}\right)-3 S_{-5} \\
-2 S_{1}^{2}\left(3 S_{-3}+S_{3}-2 S_{-2,1}\right)-S_{2}\left(S_{-3}+S_{3}-2 S_{-2,1}\right) \\
-S_{1}\left(8 \bar{S}_{-4}+\bar{S}_{-2}^{2}+4 S_{2} \bar{S}_{-2}+2 S_{2}^{2}\right) \\
-S_{1}\left(3 S_{4}-12 \bar{S}_{-3,1}-10 \bar{S}_{-2,2}+16 \bar{S}_{-2,1,1}\right)
\end{gathered}
$$

\section{Relations between weak and strong coupling regimes}

The asymptotics of $\gamma_{u n i}$ for $N=4 \mathrm{SUSY}$ at $j-1=\omega \rightarrow 0$

$$
\gamma_{u n i}^{N=4}(j)=\hat{a} \frac{4}{\omega}-32 \zeta_{3} \hat{a}^{2}+32 \zeta_{3} \hat{a}^{3} \frac{1}{\omega}-\frac{16 \hat{a}^{4}}{\omega^{4}}\left(32 \zeta_{3}+\frac{\pi^{4}}{9} \omega\right)+\ldots
$$

is in an agreement with the predictions from the BFKL equation [13].

Near the negative even points $j+2 r=\omega \rightarrow 0$ the anomalous dimension satisfy the equation

$$
\begin{gathered}
\omega \gamma_{u n i}=\gamma_{u n i}^{2}+16 \hat{a}^{2}\left(S_{2}+\zeta_{2}-S_{1}^{2}\right) \\
+4 \hat{a}\left(1-\omega S_{1}-\omega^{2}\left(S_{2}+\zeta_{2}\right)+\gamma^{2}\left(S_{2}+S_{-2}\right)\right)
\end{gathered}
$$

resumming the double logarithmic terms $\sim \alpha / \omega^{2}$ and corrections to them.

Further, the universal anomalous dimension at large $j$

$$
\gamma_{u n i}^{N=4}=a(z) \ln j, z=\frac{\alpha N_{c}}{\pi}=4 \hat{a}
$$

can be found from our results up to three loops

$$
a(z)=-z+\frac{\pi^{2}}{12} z^{2}-\frac{11}{720} \pi^{4} z^{3}+\ldots
$$

It is remarkable, that using the AdS/CFT correspondence [18] between the superstring model on the anti-de-Sitter space and the $N=4$ supersymmetric Yang-Mills theory A. Polyakov with collaborators found the coefficient $a(z)$ in the strong coupling limit [19]

$$
\lim _{z \rightarrow \infty} a(z)=-z^{1 / 2}+\frac{3 \ln 2}{4 \pi}+\ldots .
$$


In Ref. [15] the resummation of $a(z)$ in the form

$$
\widetilde{a}=-z+\frac{\pi^{2}}{12} \widetilde{a}^{2} .
$$

was suggested. The prediction of this equation

$$
\widetilde{a}=-z+\frac{\pi^{2}}{12} z^{2}-\frac{1}{72} \pi^{4} z^{3}+\ldots
$$

is in a rather good agreement with $a(z)$ in three loops and the strong coupling asymptotics.

\section{Beisert-Eden-Staudacher equation}

Our results agree also with the recent papers [20,21], where $a(z)$ is constructed in all orders. One can rewrite the Eden-Staudacher integral equation [20] as a set of linear equations [22]

$$
\begin{gathered}
a_{n, \epsilon}=\sum_{n^{\prime}=1}^{\infty} K_{n, n^{\prime}}(\epsilon)\left(\delta_{n^{\prime}, 1}-a_{n^{\prime}, \epsilon}\right), K_{n, n^{\prime}}(\epsilon)= \\
2 n \sum_{R=0}^{\infty}(-1)^{R} \frac{2^{-2 R-n-n^{\prime}}}{\epsilon^{2 R+n+n^{\prime}}} \zeta\left(2 R+n+n^{\prime}\right) \frac{\left(2 R+n+n^{\prime}-1\right) !\left(2 R+n+n^{\prime}\right) !}{R !(R+n) !\left(R+n^{\prime}\right) !\left(R+n+n^{\prime}\right) !}
\end{gathered}
$$

where the function $a(z)$ is expressed in terms of $a_{1, \epsilon}$

$$
a(z)=\frac{2\left(1-a_{1, \epsilon}\right)}{\epsilon^{2}}, \epsilon=\frac{1}{g \sqrt{2}} .
$$

We can easiliy prove, that the maximal transcendentality property for $a(z)$ is valid in all orders of perturbation theory and the coefficients in front of the products of the corresponding $\zeta$-functions are integer numbers [22]. Also $a(z)$ has an essential singularity in the point $g=\infty$.

It is possibly to show [22], that the asymptotic behaviour of $a(z)$ in the case of the BeisertEden-Staudacher equation [20] is in an agreement with the AdS/CFT prediction [19]

$$
\lim _{g \rightarrow \infty} \gamma_{s i n g}=\frac{2}{\epsilon} \frac{I_{1}\left(2 \epsilon^{-1}\right)}{I_{0}\left(2 \epsilon^{-1}\right)} \approx 2 \sqrt{2} g-\frac{1}{2} .
$$

\section{Universal anomalous dimension in 4 loops}

With the use of the asymptotic Bethe ansatz and the maximal transcendentality hypothesis the anomalous dimension in four loops was calculated [24]

$$
\begin{gathered}
\frac{\gamma_{4}}{256}=\mathbf{4} \mathbf{S}_{-\mathbf{7}}+\mathbf{6 S}_{\mathbf{7}}+2\left(S_{-3,1,3}+S_{-3,2,2}+S_{-3,3,1}+S_{-2,4,1}\right) \\
+3\left(-S_{-2,5}+S_{-2,3,-2}\right)+4\left(S_{-2,1,4}-S_{-2,-2,-2,1}-S_{-2,1,2,-2}\right. \\
\left.-S_{-2,2,1,-2}-S_{1,-2,1,3}-S_{1,-2,2,2}-S_{1,-2,3,1}\right)+\ldots \ldots \ldots \ldots \ldots \ldots \ldots \ldots \ldots \ldots \ldots \ldots \ldots \\
\quad-72 S_{1,1,1,-4}-80 S_{1,1,-4,1}-\zeta(\mathbf{3}) \mathbf{S}_{\mathbf{1}}\left(\mathbf{S}_{\mathbf{3}}-\mathbf{S}_{-\mathbf{3}}+\mathbf{2} \mathbf{S}_{-\mathbf{2 , 1}}\right),
\end{gathered}
$$


where the dots mean the dropped terms. The harmonic sums here depend on the parameter $M=j-2$. They can be analytically continued to the complex values of $M$. It turns out, that the first two terms lead to the singularity of $\gamma_{4}$ at $\omega=M+1 \rightarrow 0$

$$
\lim _{M \rightarrow-1} \gamma_{4}(M)=-\frac{512}{\omega^{7}},
$$

which contradicts to the above BFKL prediction

Therefore the asymptotic Bethe ansatz is not correct and it is needed to take into account the so-called wrapping effects. As an attempt to find the contribution of these effects we can modify the last term in the expression for $\gamma_{4}$ corresponding to the so-called dressing phase, which was found in Ref. [21] for the cusp anomalous dimension. The simplest modification conserving the transcendentality property and reproducing the BFKL prediction is given by the substitution of the factor $\zeta(3)$ in the dressing phase by the following linear combination of the harmonic sums

$$
\zeta_{3} \rightarrow \frac{47 \zeta_{3}}{24}-\frac{S_{-3}}{4}+\frac{3 S_{-2} S_{1}}{4}+\frac{3 S_{1} S_{2}}{8}+\frac{3 S_{3}}{8}+\frac{S_{-2,1}}{6}-\frac{17 S_{2,1}}{24} .
$$

It turns out, that after this substitution the anomalous dimension has the correct singularity at even negative points $M$ predicted by the double-logarithmic resummation

$$
\left.\frac{1}{256} \gamma_{4}\right|_{j \rightarrow-2 k+\omega}=\frac{5}{\omega^{7}}-20 \frac{S_{1}}{\omega^{6}}+\frac{24 S_{1}^{2}-14\left(S_{2}+\zeta_{2}\right)+4\left(S_{2}+S_{-2}\right)}{\omega^{5}}+\ldots .
$$

Therefore it is plausible, that this substitutions leads to the correct expression for $\gamma_{4}$.

\section{Discussion}

The high energy theory in QCD is based on the fact, that the gluons and quarks are reggeized. To solve the unitarization problem for the BFKL Pomeron one should use the effective action local in the particle rapidities. The Reggeon calculus in the form of a $2+1$ field theory can be derived from this action. In particular next-to-leading corrections to the BFKL kernel are calculated in QCD and in N=4 SUSY. In N=4 SUSY the eigenvalue of the kernel is expressed as a sum of the most complicated functions which could appear in this order. Using the hypothesis of the maximal transcendentality for the universal anomalous dimension of the twist-2 operators we calculated it up to the third order. Our resummation procedure is in an agreement with the strong coupling predictions obtained from the AdS/CFT correspondence. In particular, we calculated the intercept of the BFKL pomeron in $N=4$ SUSY and the cusp anomalous dimension at strong couplings. The anomalous dimension in 4-loops was also found with the use of the asymptotic Bethe ansatz, maximal transcendentality and predictions obtained from the BFKL and doublelogarithmic resummation.

\section{References}

[1] V.S. Fadin, E.A. Kuraev, L.N. Lipatov, Phys. Lett. B 60 (1975) 50; Ya.Ya. Balitsky, L.N. Lipatov, Yad. Fiz. 28 (1978) 1597.

[2] L.N. Lipatov, Phys.Lett. B 309 (1993) 394. 
[3] L.N. Lipatov JETP 90 (1986) 1536.

[4] L.N. Lipatov Phys.Lett. B 251 (1990) 284.

[5] J. Bartels, Nucl.Phys B 175 (1980) 365;

J. Kwiecinski, M. Praszalowicz, Phys.Lett.B 94 (1980) 413.

[6] L.N. Lipatov,Nucl.Phys.B 548 (1999) 328.

[7] L.N. Lipatov High energy asymptotics of multi-colour QCD and exactly solvable lattice models, Padova preprint DFPD/93/TH/70, hep-th/9311037, unpublished.

[8] L.N. Lipatov, JETP Lett. 59 (1994) 596;

L.D. Faddeev, G.P. Korchemsky, Phys. Lett.B 342 (1995) 311.

[9] L.N. Lipatov, Nucl.Phys. B 365 (1991) 614.

[10] L.N. Lipatov, Nucl.Phys. B 452 (1995) 369.

[11] E. Antonov, I. Cherednikov, E. Kuraev, L. Lipatov, Nucl.Phys. B 721 (2005) 111.

[12] V. Fadin, L. Lipatov,Phys. Lett B 429 (1998) 127;

M. Ciafaloni, G. Camici,Phys. Lett B 430 (1998) 349.

[13] A. Kotikov, L. Lipatov, Nucl.Phys. B 58219 (2000), Nucl.Phys. B 661 (2003) 19.

[14] L. Lipatov, talk at "Perspectives in Hadronic Physics", Proc. of Conf. ICTP. Triest, Italy, May 1997.

[15] A. Kotikov, L. Lipatov, V. Velizhanin, Phys. Lett.B 557 (2003) 114.

[16] S. Moch, J.A.M. Vermaseren, A. Vogt, Nucl.Phys. B 688 (2004) 101.

[17] A. Kotikov, L. Lipatov, A. Onishchenko, V. Velizhanin, Phys. Lett.B 595 (2004) 521;

Phys. Lett.B 632 (2006) 754.

[18] J.M. Maldacena, Adv. Theor. Math. Phys. 2 (1998) 231.

[19] S.S. Gubser, I.R. Klebanov, A.M. Polyakov, Nucl.Phys.B 636 (2002) 99.

[20] B. Eden, M. Staudacher, J. Stat. Mech. 0611 (2006) P014.

[21] N. Beisert, B. Eden, M. Staudacher, J. Stat. Mech. 0701 (2007) P021.

[22] A. V. Kotikov, L. N. Lipatov, Nucl. Phys. Nucl.Phys.B 769 (2007) 217.

[23] R.C. Brower, J. Polchinski, M.J. Strassler, C-I Tan, preprint hep-th/0603115.

[24] A.V. Kotikov, L.N. Lipatov, A. Rej, M. Staudacher, V.N. Velizhanin, preprint hepth/0704.3586. 